Research Article

\title{
Improvements on Photovoltaic Cells Test Bench System
}

\author{
P. N. Borza*, D. T. Cotfas, P. A. Cotfas and A. Pologea
}

Transilvania University of Brasov, Romania

Received 5 August 2012; Revised 28 September 2012; Accepted 10 October 2012

\begin{abstract}
The improvements of a test bench system for testing the solar cells is presented. The system allows measuring the I-V characteristics at different levels of lighting. Also the dynamic behavior of solar cells using light or current pulses is presented.
\end{abstract}

Keywords: photocatalysis, silver nanoparticles, methyl orange, silver-titania photocatalysis

\section{Introduction}

Nowadays the renewable energy plays a very important role. There are some types of renewable energy which were successfully implemented: the solar thermal energy, the wind energy, the photovoltaic energy, the biomass, the hydropower energy and others. The photovoltaic energy became one of the most important types of renewable energy The efficiency of photovoltaic systems depends primarily on the solar cell efficiency and the cell selection in function of its parameters. The latter has a very important influence for the lifetime of photovoltaic panels.

A system which allows determining quickly and accurately the solar cell parameters will ensure a good selection of the cells and will ensure the conditions to build a very good and reliable photovoltaic panel.

The determination of photovoltaic cells parameters was, is and will be a domain of interest for researchers. As now there are many types of photovoltaic cells it is necessary to find the suitable methods and solar cell testers which allow determining the important parameters of the solar cell: the photo generated current, the series and shunt resistances, the maximum power, the ideality factor of diode, the reverse saturation current, the fill factor and the efficiency.

There are some measurement systems which allow determining the parameters of photovoltaic cells [1-2].

To realize the measurements the test bench system for solar cells must contain: the light source, the I-V characteristic module, the thermostat, the mobile part, the acquisition and control system and the dedicated software. In industrial applications, the solar simulator is built using halogen lamps, even if the matching between the spectrum of the halogen lamp and the solar spectrum is not very good. This happens due to the lower cost on the illuminated surface [1]. The module for measuring I-V characteristics has to permit the measurement in a very short time (seconds), and the number of measurement points has to be large enough to allow a good data processing.

Characteristics' measurement can be done through various techniques: the use of an electronic load, of a capacitor as a variable load, of a MOSFET or a digital potentiometer [3]. The variation of the illumination levels can be done by varying the distance between the solar cell and the light source. A thermostat can be used to maintain the temperature constant during the measurement time. The levels of illumination can be determined using the photo detectors.

\section{Experimental \\ Experimental set-up}

The architecture of the test bench system is based on the integration of three parts: the mechanical sub-system, optical sub-system and electronic sub-system. The first one ensures the motorization of the optical sub-system that implements the variation of light flow on the DUT. The quality of implementation is related to the following factors: precision of the positioning system, stabilization of light flow provided by the light source and the data acquisition system [4]. In the first phase, the solar cells characterization system was developed using a SDK ATmega system and a data acquisition board NI 6215 that together with a personal computer ensured the local control of the test bench [4].

The test bench developed for characterization of photovoltaic cells is presented in Fig.1.

\footnotetext{
*E-mail address: borzapn@unitbv.ro

ISSN: 1791-2377 @ 2012 Kavala Institute of Technology. All rights reserved.
} 


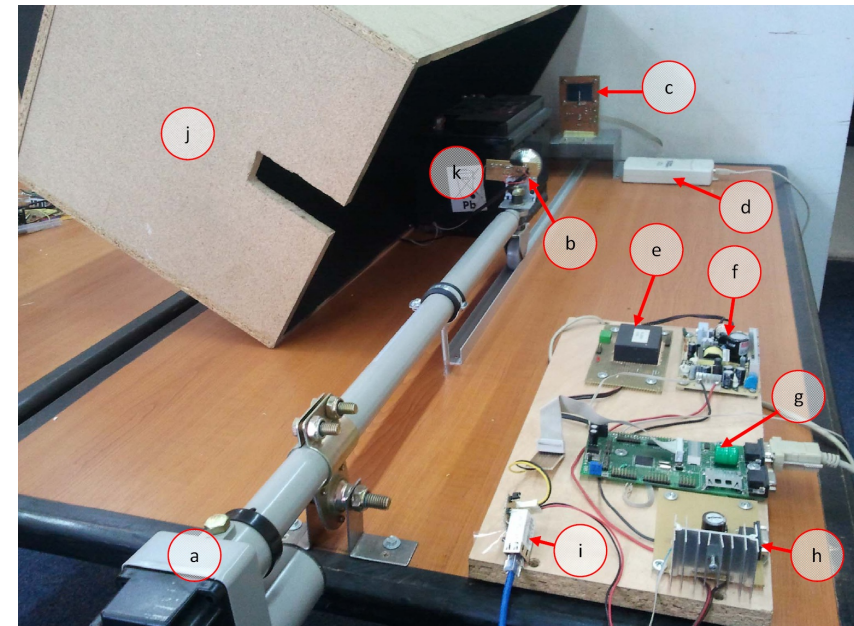

Fig. 1. The test bench system for characterizing the photovoltaic cells

Its structure comprises of the following [4]:

a) Linear actuator - permits the movement of the light source in order to obtain a variable lighting level. The movement is realized on $50 \mathrm{~cm}$, ensuring a irradiance variation from $100 \mathrm{~W} / \mathrm{m} 2$ until 2000 $\mathrm{W} / \mathrm{m} 2$.

b) Light source - is a halogen light bulb with an aluminium reflector assuring a light spot with a high uniformity and the switcher on/off based on a power MOSFET.

c) Device under test, DUT - formed by the monocrystalline silicon photovoltaic cell with the dimensions $3 \mathrm{~cm} / 5 \mathrm{~cm}$ (Fig. 2 a), 4 photoresistors placed in the corners of the cell (Fig.2) a temperature sensor (LM35 case TO46) placed on the back of photovoltaic cell.

d) The data acquisition board NI USB 6215 is used to measure the I-V characteristics of the photovoltaic cell, the signals on the four photoresistors and the signals given by the temperature sensor, and also to command the MOSFET used as electronic load for the photovoltaic cell;

e) The power source of $5 \mathrm{~V} \mathrm{DC}$ - used to power the SDK ATmega system;

f) The power source of $12 \mathrm{~V} \mathrm{DC}$ - used to power the linear actuator;

g) SDK ATmega system - used to control the actuator and switch on/off the light source;

h) $\mathrm{H}$ bridge driver to command the linear actuator;

i) Embedded server Lantronix - permits the access to SDK ATmega system over the network;

j) Box - used to ensure the lighting of the solar cell only by the light source used and eliminate all other light sources in the lab;

k) $12 \mathrm{~V}$ batteries - used as a power source for the light source. This ensures a constant voltage of the light source eliminating the fluctuations existent at the power sources DC $(10 \mathrm{~Hz})$.

In the second phase, the characterizing system of the photovoltaic cells was modified for simplification purposes [4]. The system's structure was modified by replacing the data acquisition board, NI 6215, with the SDK ATmega system to which a module was added formed by two digital synchron converters endowed with programmable amplifiers $[6,7]$.

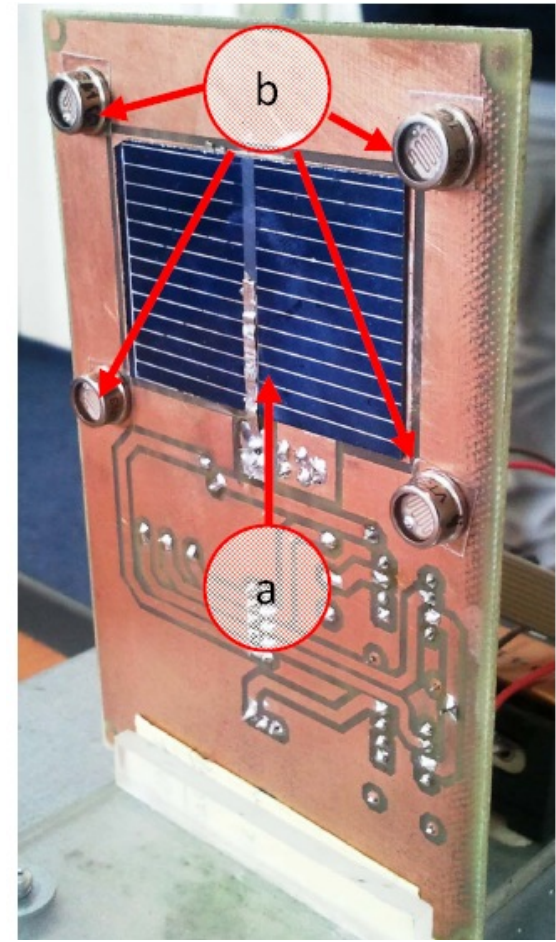

Fig. 2. The device under test

\section{Methodology}

The new system has used the same set-up like the old that was described above. During the measurement process the temperature of sensor and PV cells slightly vary in the interval that of temperature sensor resolution (less than $1.5^{\circ} \mathrm{C}$ ). Thus, the duration of a testing process is less than $2 \div 3$ seconds and we have considered the temperature variation as not significant. We mention that the case used for temperature sensor assure a time constant around 1s and the thermal resistance between PV cell and sensor was minimize by using a special very low resistance gel material. The performance of the designed system was improved [4] by increasing the sampling rate and as result, the throughput of data transfer for the signal acquired. In the same time using a PGA (LMS8353), with zero drift (automatically compensated) and facilities to auto-detect his own status, in conjunction with the designed software, was designed a set of routines that permit the implementation of a self healing reaction from our improved system. Bellow are described the means that have assured these functionalities.

The data acquisition from AD161S626 suppose to provide 18th clock pulse in order to assure the data acquisition; see Fig. 3 [7].

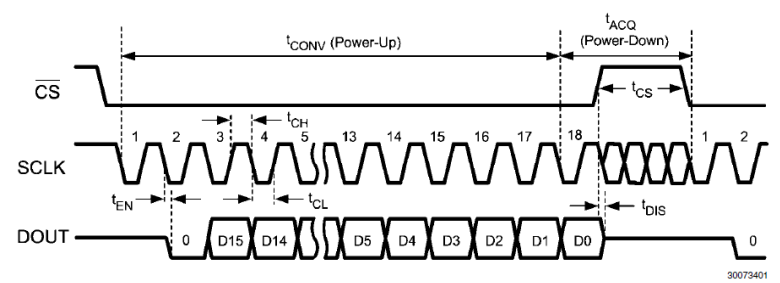

Fig. 3. Timing for data acquisition $n$ case of ADC 161S626 used as analog to digital converter $[7$

The first two pulses are used internally to provide the sampling of input analog signal and the second pulse provides the frame. The next 16th pulses assure the 
extraction of data from AD converter to the SPI interface. The initial routine was used for this acquisition of $3 \times 8$ clock pulses -MSB, NSB and LSB bytes, see Fig. 4 and also has performed the re-alignment of the data on two bytes. Thus, supposing that the SPI clock is $3.618 \mathrm{MHz}$ the period is $276 \mathrm{~ns}$, respectively the transfer of 8 th bits consume $2.21 \mu \mathrm{sec}$. In order to provide the data for the test bench supervisor, the information acquired was rearranged by several successive instructions: $\mathrm{MSB}<<2 \mid(\mathrm{NSB}>>6$ \& $0 \mathrm{x} 03), \mathrm{NSB}<<2 \mid(\mathrm{LSB}>>6 \&$ \& 0303$)$ obtaining the two bytes corresponding at the input converted signal. In this case the time consumed for acquisition was:

$$
\tau_{\text {old }}=13.40 \mu \mathrm{s}
$$

The improved solution was designed by exploring the dynamic exchange of SPI settlements. In the first step for the first two clock pulses the interface was settled as GPIO and using software pulse generation was provided the signals necessary to assure the sampling of the signal and the start bit. Afterward, the interface was re-programmed as SPI and 2 bytes was acquired. These bytes contain the numerical values corresponding at signal conversion, Fig.5.

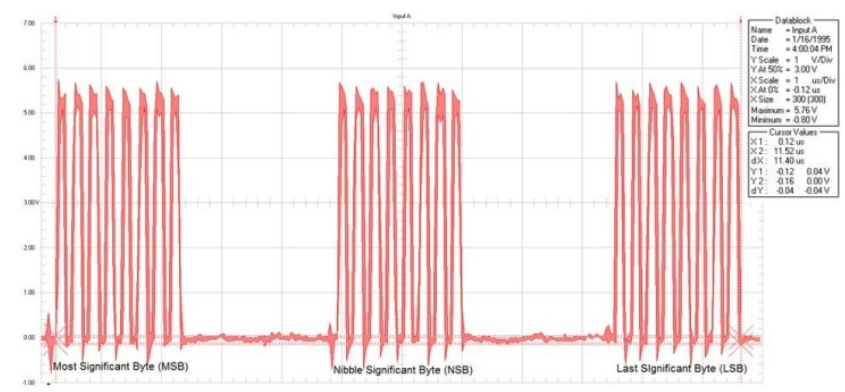

Fig. 4. Timing of signal acquisition using event drive routine (Interrupt service routine-only)

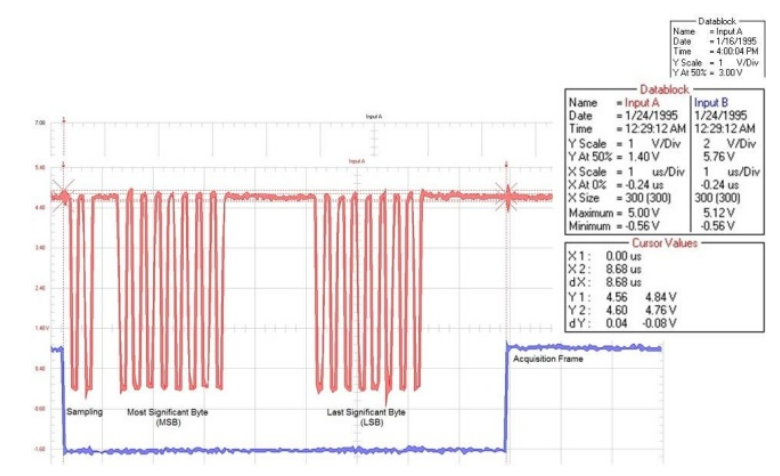

Fig. 5. Timing of signal acquisition using mixed software solution (programmed \& event drive routine (Interrupt service routine-only))

A simple calculus is given by the relation illustrated bellow. This formula also takes into account the execution time for re-programming the SPI interface $\tau_{\text {new }}=8.68 \mu \mathrm{s}$. The reduction in data acquisition time is $35.22 \%$. This consequence is only the result of using of the SPI interface for transfer of digital data between ADC and microcontroller system. The supplementary instruction necessary in the first situation means a spent time of $0.414 \mu \mathrm{s}$, respectively in the end the savings represents: $37.16 \%$.
The second improvement is related to the modification of firmware that assures the auto detection of self status for PGA. Thus, the acquisition chain was periodically calibrated by applying on the input acquisition channel the voltage reference and calculating, on the basis of this value, the corrected data. Thus, the slight variation of voltage reference during long term acquisition was taken into account. Using these facilities the precision of the acquired data was improved. Moreover, by software the inputs of PGA were switched on PGA internal current sources and thus the eventual wiring faults on the system inputs could be detected. Thus, the input circuitry of our test bench is periodically verified by the program. This facility was implemented because normally the duration of the test, especially for aging test takes a very long time and the integrity of the test bench system is mandatory to be revalidated.

\section{Conclusions}

The test bench system is a compact and flexible system to characterize the photovoltaic cells.

The measurements can be performed manually and automatically, allowing the user to adapt his strategy at the specific of PV cells tests requirements.

The test bench assures a very precise calibration for the level of irradiance in function of the distance between the light source and the photovoltaic cell and allow the permanent compensation of lighting variation of PV cells.

The price of the test bench photovoltaic cell is very low, making it suitable for the market.

The photovoltaic cells are characterized using I-V characteristics. These characteristics are measured at different levels of lighting.

Related to the high level software this is able in the new version to generate in real time the drawing of $\mathrm{I}-\mathrm{V}$ characteristics of photovoltaic cells and to store and calculate the statistic parameters for the series of test done. Thus, the improved solution represents a complete and accurate system suitable to be used for the characterization of all types of photovoltaic cells. Silver can be deposited on the surface of $\mathrm{TiO}_{2}(\mathrm{P} 25)$ using aqueous AgNO3 solution and a UV photocatalytical process. The increase of $\mathrm{AgNO}_{3}$ content in the solution, results in higher $\mathrm{Ag}$ amounts in the structure. Photocatalytical decomposition of methyl orange by $\left(\mathrm{Ag} / \mathrm{TiO}_{2}\right)$ depends on $\mathrm{Ag}$ content in the structure. Elevated Ag content brings to higher rate of methyl orange decomposition. The probable mechanism might be recombination of UV irradiation induced electrons and holes. As a result the rate of methyl orange decomposition on $\left(\mathrm{Ag} / \mathrm{TiO}_{2}\right)$ substantially grows as compared to the standard substrate P25. High photocatalytic activity for the $\mathrm{MeO}$ reduction using lamps of low energy UV emission $(\lambda=$ $365 \mathrm{~nm})$ was obtained for the $\left(\mathrm{Ag} / \mathrm{TiO}_{2}\right)$-catalysts, improving the rate constant of reduction with up to 3 times compared to $\mathrm{TiO}_{2}$ alone (reference). These results evidence a very important role of $\mathrm{Ag}$ in photoreduction reactions for organic compounds, in this particular case for the reduction of $\mathrm{MeO}$. The methyl orange photocatalytic decomposition corresponds to a first order reaction. These catalysts exhibit a significant efficiency by the methyl orange decomposition even under solar irradiation only. The physical methods used show the presence of a silver phase (TEM), a polycrystalline structure (SAEDI), and lack of change of the structure and composition of modified with silver $\mathrm{TiO}_{2}$ (XRD). 
As conclusion, the reported results indicate that $\left(\mathrm{Ag} / \mathrm{TiO}_{2}\right)$-catalysts can be successfully used for the complete degradation of azo-dyes.

\section{References}

1. G. Aberle, T. Lauinger, S. Bowden, S. Wegener, G. Betz, Photovoltaic Specialists Conference, Conference Record of the Twenty Fifth IEEE, pp. 593 - 596 (1996).

2. M. A. Mosalam Shaltouta, M. M. El-Nicklawyb, A. F. Hassanb, U. A. Rahomaa, M. Sabrya, Renewable Energy, 21, 445 (2000).

3. D. T. Cotfas, P. A. Cotfas, D. Ursutiu, C. Samoila, 12th International Conference on Optimization of electrical and electronic equipment OPTIM 2010, May 20-22, pp. 1115-1120 (2010).

4. P. N. Borza, P. A. Cotfas, D. T. Cotfas, M. C. Carp, 13th International Conference Optimization of Electrical and Electronic Equipment (OPTIM), pp. 1046-1051 (2012)

5. A. S. Moraru, M. Romanca, P. Borza, The $2^{\text {nd }}$ International Workshop on e-learning and Virtual and Remote Laboratories
2008, February 14-15, 2008, Hasso-Plattner-Institute Potsdam, Germany, pp 21-28 (2008).

6. F. Sandu, M. Romanca, A. Nedelcu, P. Borza, R. Dimova, Proceedings of „OPTIM 2008”-11th International Conference on Optimization of Electrial and Electronic Equipment, pp. 225-230 (2008).

7. ADC161S626 16-Bit, 50 to $250 \mathrm{kSPS}$, Differential Input, MicroPower ADC - SNAS468B", Texas Instruments, Post Office Box 655303, Dallas, Texas 75265, 2011Chin-Shun Tsai, ChengTao Hsieh, and Shyh-Jier Huang "Enhancement of DamageDetection of Wind Turbine Blades Via CWT-Based Approaches" IEEE transactions on Energy Con version,Vol. 21, No. 3, Sept. 2006. pp 776-781. 